\title{
A New Algorithm of Eyed Typhoon Automatic Positioning Based on Single Infrared Satellite Cloud Image
}

\author{
Gang Yin ${ }^{1}$ \\ School of Measurement-Control and Mechanical- \\ Electrical Engineering \\ Inner Mongolia Vocational College of Chemical \\ Engineering \\ Hohhot, China \\ e - mail: chenyufeng1964@163.com
}

\author{
Yu Feng Chen ${ }^{2}$ \\ School of Mechanical and Electrical Engineering \\ Hohhot Vocational College \\ Hohhot, China \\ e- mail: chenyufeng1964@163.com
}

\begin{abstract}
According to the characteristic that the gray value difference is quite obvious in different areas of the singles infrared cloud, this paper puts forward an algorithm of the eyed typhoon's center automatic positioning. After pretreating the cloud image, we use the $3 \times 3$ template to segment the airtight cloud wall area which has high gray value. In addition, the difference between gray values of the airtight cloud wall area is small. Then we do binarization processing to the image and use the method combined with the mathematical morphology and artificial intelligence to process the image in filtering smoothly. We remove the isolated interference point. Then we corrode image and adopt the seed filling algorithm to detect the candidate eye area. In the candidate eye area, the paper puts forward the discriminant function which is based on the combination of the gray information and the distance between the inspection points and the circle's center. The candidate eye area corresponded to the minimum value of the discriminant function is the center of the eyed typhoon. The experiment result shows that this algorithm has higher positioning accuracy and it can be used for meteorological service.
\end{abstract}

Keywords-the singles infrared cloud; the center of the eyed typhoon; automatic positioning; mathematical morphology

\section{INTRODUCTION}

In the cloud images of several different band channels transferred by the GMS-5 geostationary satellite[1], the infrared channel cloud picture's gray level are much rich, about 256 ranks, which reflects the absolute temperature of corresponding points. For the difference among the gray value of different parts of the cloud picture, the automatic locating of eyed typhoon center could be achieved by the method based on single infrared image. Typhoon can be divided into eyeless typhoons and eyed typhoon which has confined cloud wall, spiral cloud bands and eye area. When the tropical cyclone eye area is small or the vortex center is quite clear, the cyclone eye or the vortex center can be regarded as the center of the typhoon. If the eyes are large and round, then the geometric center of the tropical cyclone can be regarded as a typhoon center; and if eyes are large and irregular, then the warmest point of the infrared cloud eye area can be regarded as the center of the typhoon.

\section{Cloud Picture Pretreating}

Before designing the typhoon positioning algorithm, in processing of the original satellite image must be taken aim at eliminating noise and extracting the target cloud system [2].

\section{A. Removing Image Noise}

There are various kinds of noise existing in the original GMS-5satellite cloud picture, mainly including scanning noise and pepper noise, which seriously affect the positioning accuracy, and in order to eliminate the effects of noise, image smoothing processing must be carried out. In this paper, the author used a simplified "pseudo median filtering method" [3], selecting a moving window of $3 \times 3$ and taking the values in the first three rows of elements, then taking the median of three values. The filtering effects are shown in Fig 1 and Fig. 2. In Fig. 1, the salt and pepper noise are completely removed.

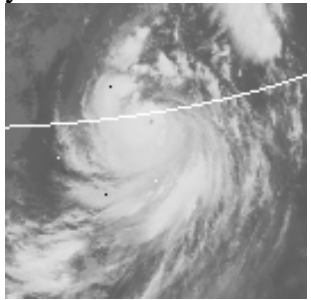

Figure 1. Original cloud image including noise

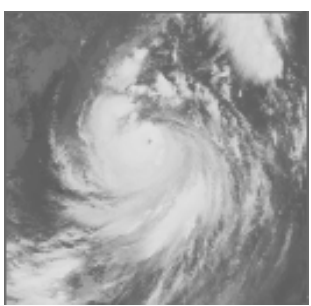

Figure 2. Cloud image after filtering

\section{B. Segment the Typhoon Cloud}

It is a prerequisite for locating the center of Typhoon Cloud that extracts cloud system from the original cloud 
image. A cloud image often contains a variety of different cloud systems whose gray values vary with cloud system types, geographical and time. And meanwhile, for the quite irregular shape of the cloud system, we can not simply split different cloud systems by the difference between thresholds or shapes [4, 5]. In this paper, the author uses the mathematical morphology multi value segmentation method to split different cloud systems. The core of the algorithm is to generate an adaptive template based on the morphological characteristics of infrared satellite cloud image and the template includes image segmentation information so that we can extract the cloud picture with larger area and low temperature. The method can be divided into four steps:

1) Divide the cloud picture into regions:

First we locate the cloud picture maximum pixel gray level and the central region' s gray level, and then determine each of the center region of the cloud system with a threshold determined by the gray level of center region. And divide these points in accordance with the distance between the pixel areas so we can obtain the amount of split parts and the center point of every split part.

2) Corrode every region and take "and" operation between corrosion treatment results and the center of each divided region:

We corrode every region by using appropriate elements and then take "and" operation between corrosion treatment results and the center of each divided region, and note the time of the corrosion times and the corresponding divided regions to obtain each cloud system initial split template, the corrosion number and corresponding structural elements.

3) Conduct a dilation operation on the center of each divided region:

Conduct a dilation operation on the center of each divided region by using 2) with the corresponding structural elements and frequency, then taking a merge operation with the initial segmentation template in 2), to obtain the adaptive segmentation of the various regions of the template.

4) Opening and closing operation treatment

We take "and" operation between adaptive split template and preliminary segmentation of images so that we can extract each main cloud system from the satellite image segmentation approximately. Then we can get split image using smooth opening based on opening and closing operations of mathematical morphology.

\section{The Eyed TYPHOON POSITIONING AlgORITHM}

In reference [4],the main method of eye point recognition is achieved by calculating and judging the maximum temperature, average temperature of each cloud hole in the cloud wall, as well as the minimum temperature and average temperature of cloud -dong peripheral cloud band on ( about $55 \mathrm{~km}$ Department ) the annular envelope line. However, this method can not identify the eye area of diameter more than $40 \mathrm{~km}$ or less than $10 \mathrm{~km}$.

The eye area of eyed typhoon is surrounded by a cloud area with a large area with a quite low temperature. However, the eye area itself has a relatively high temperature. Besides the airtight wall of eyed typhoon is an area with high gray value and the adjacent pixel gray value close to the area. While the gray value of pixel is quite different between the eye part and its adjacency. Therefore, it is a feasible method to judge the eye area by looking for the highest temperature point in the area whose radius is airtight wall's center. The method used in this paper can be generally summarized as the following three points:

1) Mature typhoon cloud has obvious eye area, closed wall cloud and background;

2) Airtight cloud wall gray value is very high and close to the adjacent pixel's, while luminance is brighter than the corresponding background and other cloud blocks and the temperature is lower. Meanwhile, it is quite different from the eye part gray value. We can extract the eye area using intelligent threshold that is used to separate the airtight cloud wall;

3) The eye part of eyed typhoon is located at the center of airtight cloud wall where the temperature is the highest.

With Numbers \# 99092210 typhoon cloud image as an example, the algorithm can be divided into six steps:

1) Split out airtight wall cloud

Design a $3 \times 3$ template like fig3 below. $P$ is the gray value of template center pixel and $P_{i}$ is the 8 neighborhood gray value, for $i=0,1 \cdots 7$.

\begin{tabular}{|c|c|c|}
\hline$P_{3}$ & $P_{2}$ & $P_{1}$ \\
\hline$P_{4}$ & $P$ & $P_{0}$ \\
\hline$P_{5}$ & $P_{6}$ & $P_{7}$ \\
\hline
\end{tabular}

Figure 3. 3x3 template

This template traverses the entire cloud picture area from left to right, from top to bottom. In the process we calculate the absolute of the difference between the center pixel's gray values and eight neighborhood's gray values, namely

$$
\Delta P_{i}=\left|P-P_{i}\right|
$$

Then find the maximum of eight difference value above $\max \left(\Delta P_{i}\right)$.If $\max \left(\Delta P_{i}\right)$ is less than segmentation threshold $L_{0}$ and center pixel gray value is greater than the segmentation threshold $K_{0}$, the pixels belongs to the closed wall cloud area, namely: the center pixel gray value set 0 . Written rules for:

$$
\text { If }\left|\max \left(\Delta P_{i}\right)\right|<L_{0} \text {, and } P>K_{0} \text {, then } p=0 \text {; }
$$

Thus we split out the closed wall cloud area. $L_{0}=12, K_{0}=200$, Numbers \# 99092210 typhoon cloud image processing results is shown in Fig. 4. 


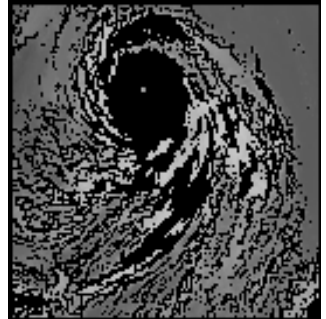

Figure 4. Cloud image after airtight cloud wall extracting

\section{2) Image binarization}

Binarization function can be described as

$$
f(g)= \begin{cases}b_{1} & g<\mathrm{d} \\ b_{2} & \mathrm{~g} \geq \mathrm{d}\end{cases}
$$

Here, $0 \leq b_{1}, b_{2} \leq 255$, and in this function $b_{1}=0, b_{2}=1$; $g$ is the gray value; $d$ is the binarization segmentation threshold obtained by experiments.

Set the pixels of the typhoon cloud background which is after binarization operation. The rest parts include background and we set the eye area pixel to 1 . The eye area which is after binary is isolated out. The result can be seen in Fig. 5

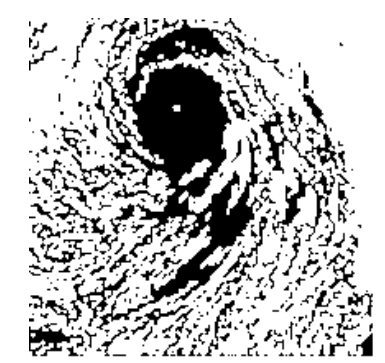

Figure 5. Cloud image in binary value codes

3) Morphological operation, image smoothing filtering processing and remove isolated interference point [6, 7]

Setting $\mathrm{F}$ is original gray cloud image, $\mathrm{B}$ the radius for $\mathrm{R}$ spherical structure elements, the open and close operation formula can be described as the following formula:

Open operation:

$$
\bigcup\left(F_{B}\right)=\bigcup(F \Theta B \oplus B) .
$$

Close operation:

$$
\bigcup\left(F_{B}\right)=\bigcup(F \oplus B \Theta B) .
$$

The result of taking binarization operation for cloud picture is shown at Fig. 6.

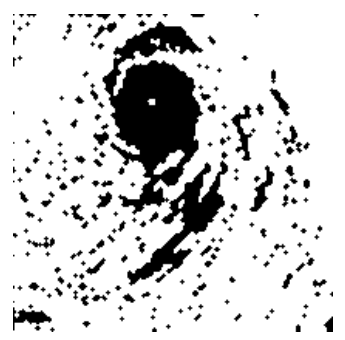

Figure 6. Image after open and close operations

After Open and close operation, expand the part whose gray value is 1 with a round structure elements with radius of 1. The result is shown in Fig. 7.

Then corrode the part whose gray value is 1 with a round structure element with radius of $2 \sqrt{2}$ twice in order to eat off isolation jamming point outside the airtight cloud wall. The first time is shown in Fig.8 and the second time in Fig. 9.

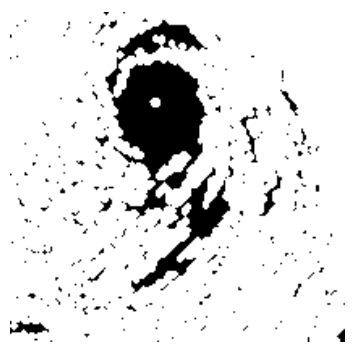

Figure 7. Image after the dilation operations

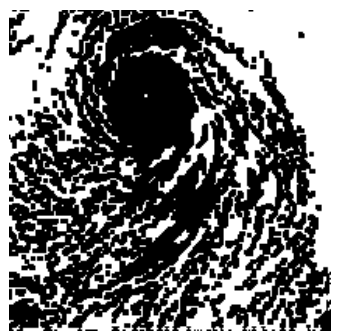

Figure 8. Image after the first erosion operations

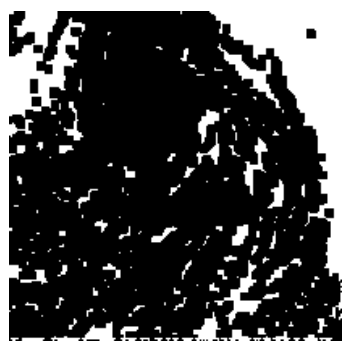

Figure 9. Image after the second erosion operations

4) Reset the cloud area remaining after corrosion to 2(the part in Fig.9 with white background) Reset the part whose gray value is 0 after corrosion to 1[8].

5) Use seed filling algorithm to detect the eye area The background is connected and the eye area is isolated, 
detect 、 mark and remove all non isolated area, so that there are only the eye area left.

a) Suppose the upper-left corner pixels of the binary image are within the region. Set their gray value to 2 .

b) Judge all the pixels point whose gray value is 2, whether there is pixel whose gray value is 1 , if there is, set its gray value to 2 .

c) Circulate 2), and judge whether the treated images and pretreated images are equal. If equal, it indicates that all the neighborhoods are filled. We exit the loop.

d) After treatment, there are only large dense cloud wall, background and candidate eye areas. Thus, the images are composed by three pixels whose gray values are 0,1and 2 respectively. The closed wall cloud area's gray value is 0 and isolated area's is 1.The rest department's gray value is 2 ;

e) Set the gray value of the department whose gray value is 2 to 255 and segment the candidate eye area, see Fig 10;Combined with the original cloud image, we have get the Fig. 10 .

f) Fig. 11 can clearly reflect the candidate eye area's exact location in the cloud image.

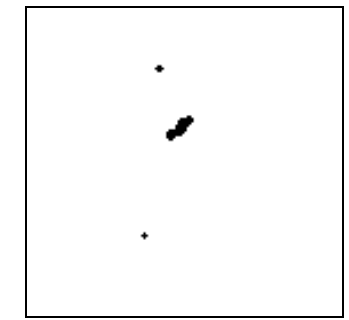

Figure 10. Eye areas segmented

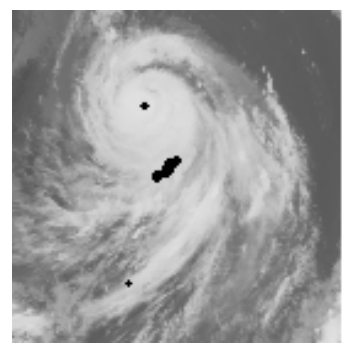

Figure 11. Image of candidate eye areas

6) Confirm the eye area of the eyed typhoon in the candidate eye areas.

The center of the airtight cloud wall is generally not typhoon's eye area. So we look for the highest point of the temperature value as the eye area in the area whose circle center is the center of the airtight cloud wall.

In Fig. 4, the area whose gray value is 0 is the airtight cloud wall and we record it as B. Then we solve the center.
Suppose $R(d)$ representing circular structure element whose radius is $d, d=35,34,33, \cdots$ (Take the initial value 35 ), the corrosion arithmetic formula is

$$
B^{\prime}=B \Theta R(d)
$$

This formula shows that it corrode $B$ with the circular structure element $R(d)$.

We reduce $d$ sequentially and take $d$ as the radius and the remaining points as the center of the circle when the corrosion results are not empty. In order to reduce the computational time, adopt the "m" Font simplified circular structure elements instead of circular structure elements and the effect is better.

Look for the eye area in the range of the cloud wall's maximum inscribed circle. Because the eye area's gray value is lower than the dense cloud wall's, so the gray value information can be used as the basis for recognizing the eye region. But in the range of the maximum inscribed circle, when there are cloud seams, cloud cave and its gray value is lower than the eye area's, we can't only take the gray value as the discriminate standard. In order to reduce the interference of cloud seams and cloud caves, when discriminating eye area, it is necessary to consider the distance between the inspected point and the circle's center. According to the meteorological experience, usually the distance between eye area and the center of the circle is smaller than the distance between the cloud seam and the center of the circle.

We use circular structure elements whose radius is three to repeatedly expand the heart area of the inscribed circle. We suppose that it expands n times to the inscribed circle's radius. Then we stop expanding and it forms $\mathrm{n}$ concentric circles area. To recognize the eye area of the eyed typhoon, as previously mentioned, the algorithm uses the sum of the gray-scale information and distance information as judgment function. The judgment formula is

$$
j u d g e(i)=\operatorname{gray}(i)+K \times I \text {. }
$$

In the formula, $i$ is the expansion number of the region, $i$ $=1,2 \cdots n ; \operatorname{gray}(i)$ is gray information; we choose the candidate eye area's gray value at the $i$ times expansion. $K$ is the radius of the expanded circular structure element. $K=3$; Use $K \times I$ to measure the distance information. The information represents the distance between the candidate eye area and the center point at the number $i$ expansion. Take the candidate eye $\min (j u d g e(i))$ as the resolved typhoon eye area.

\section{THE EXPERIMENTAL RESULTS}

The automatic positioning of the eyed typhoon's center which is based on the single cloud picture is implemented on IBMSP2 which is the massively parallel computer. Parallel algorithm adopts the static division and the dynamic 
TABle I. Locating Results OF North-Western Pacific And SOUth SeA Cyclones In 2001

\begin{tabular}{|c|c|c|c|c|c|c|}
\hline $\begin{array}{c}\text { Serial } \\
\text { Number }\end{array}$ & $\begin{array}{c}\text { Positioning } \\
\left.\text { Longitude/( }{ }^{\circ}\right)\end{array}$ & $\left.\begin{array}{c}\text { Positioning } \\
\text { Latitude/( }\end{array}{ }^{\circ}\right)$ & $\begin{array}{c}\text { Typhoon } \\
\text { Properties }\end{array}$ & $\begin{array}{c}\text { Actual } \\
\text { Longitude/( }\end{array}$ & $\begin{array}{c}\text { Actual } \\
\text { Latitude/ }\end{array}$ & $\begin{array}{c}\left.{ }^{\circ}\right) \\
\text { Typhoon } \\
\text { Name }\end{array}$ \\
\hline 01062020 & 13.40 & 129.90 & Set wrong & 14.1 & 128.7 & CHEBI \\
\hline 01062100 & 13.25 & 128.20 & Set wrong & 14.3 & 127.6 & CHEBI \\
\hline 01070112 & 19.40 & 113.58 & The eyed typhoon & 20.2 & 111.5 & DURIAN \\
\hline 01072502 & 20.73 & 114.37 & The eyed typhoon & 20.6 & 113.9 & YUTU \\
\hline 01072507 & 21.55 & 113.80 & The eyed typhoon & 27.5 & 140.5 & YUTU \\
\hline 01091108 & 26.34 & 126.76 & The eyed typhoon & 26.3 & 127.2 & NARI \\
\hline 01091112 & 26.40 & 126.91 & The eyed typhoon & 26.4 & 127.2 & NARI \\
\hline 01091404 & 27.69 & 125.31 & The eyed typhoon & 27.6 & 125.0 & NARI \\
\hline 01091519 & 25.93 & 123.61 & The eyed typhoon & 26.0 & 124.1 & NARI \\
\hline 01091610 & 25.57 & 122.89 & The eyed typhoon & 25.1 & 122.2 & NARI \\
\hline 01100711 & 25.54 & 136.38 & The eyed typhoon & 26.3 & 137.6 & KROSA \\
\hline
\end{tabular}

allocation which is based on task.

Randomly select the tropical cyclone detection system positioning situation of the northwest Pacific and South Sea, The results is shown as in TABLE I.

Since there is no objective typhoon center coordinates , In the TABLE I, typhoon's actual latitude and longitude take the typhoon center coordinates which are published by the National Weather Service. The conclusion is drawn by the meteorological workers subjectively marking in satellite cloud image according to their experience. The results show that the algorithm introduced in this paper has a high recognition rate and positioning accuracy to meet the weather business requirements.

\section{EPILOGUE}

At present, it is still in the stage of exploration both at home and abroad in using image recognition and artificial intelligence to research the localization problem of satellite cloud image and typhoon cloud system. The author used the singles infrared satellite cloud image of the GMS25 geostationary satellite, combined with meteorological domain knowledge, and proposed the center automatic positioning algorithm of the eyed typhoon system. The algorithm uses a combination of mathematical morphology and artificial intelligence to resolve the eyed typhoon center positioning problem better. The experimental result shows that the algorithm has higher positioning accuracy and it can be used for weather forecasting business. In addition, it has made beneficial exploration for the meteorological service of the typhoon center automatic positioning systems. However, the single infrared satellite cloud image has limited information. How to use multiple cloud images or multispectral image to improve the algorithm's robustness and generality needs further research.

\section{REFERENCES}

[1] Jiang Shangcheng, "The channel selection of the meteorological satellite and its application” [J]. Meteorological Monthly, 1999, 25(3), pp. 29-35.

[2] Wang Zhilie. Typhoon Forecasting Hand book [M]. Beijing: China Meteorological Press, 1987

[3] Liu Zhengguang, Lin Kongyuan. "Extracting morphological features of satellite image”. Computer Research and Development, 1997, 34 (9), pp. 689-693.

[4] Feng Minxue, YuBo, ChenBiyun, et al. "Automatic location method of the eyed typhoon”. Meteorological Monthly, 1997, 23 (12), pp.15-17.

[5] Liu Yong. "Study of Satellite Image Database" .Tianjin: School of Electrical and Automation Engineering Tianjin University, 2000 (in Chinese).

[6] SoilleP. Morphological Image Analysis.PrinciplesandAppli2 cations[M]. Berlin: Springer Verlag , 1999.

[7] Soille P, Talbot H. "Image structure orientation using mathe-matical morphology". In: Proceedings of the Fourteenth International Conference on Pattern Recognition[C]. 1998,2, pp.1467-1469.

[8] Soille P, JonesR. Recursive implementation of erosions and Dilations along discrete lines at arbitrary angles [J]. IEEE Trans on Pattern Analysis and Machine Intelligence, 1996, 18

[9] WEI K, JING Z L. "Spiral band model optimization by chaos immune evolutionary algorithm for locating tropical cyclones” [J]. Atmospheric Research, 2010, 97(1-2), pp. 266-277.

[10] WEI K, JING Z L, LI Y X, et al. "Spiral band model for locating tropical cyclone centers” [J]. Pattern Recognition Letters, 2011, 32(6), pp.761-770.

[11] WONG K., YIP C L, LI., P W. "Automatic tropical cyclone eye fix using genetic algorithm” [J]. Expert Systems with Applications, 2008, 34(1), pp.643-656.

[12] Piñeros M F, Ritchie E A, Tyo J S. “Objective Measures of Tropical Cyclone Structure and Intensity Change from Remotely Sensed Infrared Image Data" [J]. IEEE Transactions on Geosciences and Remote Sensing, 2008, 46(11), pp.3574-3580. 